\title{
LINGUA FRANCA Y LENGUA DE MOROS
}

\author{
Bruno Camus Bergareche. \\ Universidad de Castilla-La Mancha.
}

\section{INTRODUCCIón.}

El problema principal en el estudio del histórico pidgin mediterráneo conocido como lingua franca es el escaso número de testimonios documentales. Entre estos se han venido citando, por lo menos desde el artículo pionero de Hugo Schuchardt de $1909^{1}$, la jerga de moros del teatro clásico español. El propósito de este trabajo es precisamente describir y analizar la naturaleza estructural de esta lengua con el fin de precisar posteriormente su relación con la lingua franca. Hablaremos en primer lugar de esta llamada lingua franca y de los textos que de ella disponemos, entre ellos los textos en lengua de moros. Procederemos después al análisis pormenorizado de esta jerga, el contexto literario en que aparece y sus caracteristicas internas. Finalmente, nos serviremos de todos estos datos para llevar a cabo una revisión de la relación entre lingua franca y lengua de moros que a su vez sirva también para replantear la naturaleza de otros textos de parecida naturaleza, igualmente relacionados tradicionalmente con la lingua franca.

\section{La Lingua franca.}

El estudio de las antiguamente denominadas lenguas mixtas, que tuvo entre sus cultivadores primeros a Hugo Schuchardt, ha venido ganando progresivamente crédito desde que los sociolingüistas y generativistas anglosajones comenzaron a tomar interés por el tema en los primeros años sesenta. Hoy día no hay manual de Lingüistica Histórica o Sociolingüística que no

1 H. Schuchardt, "The Lingua Franca", en Pidgin and Creole Languages, Cambridge, 1980 , págs. $65-88$. 
les dedique un importante espacio y conceptos como criollo o pidgin resultan actualmente familiares. Efectivamente, el estudio de este tipo de códigos restringidos ha contribuido considerablemente en los últimos años a aumentar el conocimiento de procesos como la adquisición del lenguaje, el cambio lingüístico, el multilingüismo, etc. Cada año aumenta en varias unidades el número de pidgines y criollos catalogados, al tiempo que se amplía el número de publicaciones a ellos dedicadas. Lo que hasta hace poco se suponia era un fenómeno básicamente ligado a la expansión y colonización europeas se reconoce ya como un hecho de alcance universal y mucho más antiguo. $\mathrm{E} 1$ interés no se limita a situaciones actuales sino que, retomando el camino iniciado en su día por Schuchardt, se vuelve la vista a pidgines históricos ya desaparecidos. Es el caso de la investigación en torno a la lingua franca, investigación que, dada la naturaleza de esta lengua, entronca inevitablemente con disciplinas más tradicionales y de muy diferentes propósitos, como la Lingüistica Románica o la Filología.

La denominación lingua franca tiene en el contexto de estos estudios criollos dos acepciones muy diferentes. En primer lugar, sirve el término lingua franca para designar a todas aquellas lenguas que se usen en contextos multilingües como instrumento común de comunicación, es el caso de lenguas de uso internacional como el inglés. Sin embargo, en la acepción que nos interesa, lingua franca es el nombre que recibe un pidgin de base románica de uso habitual en la cuenca mediterránea, sobre todo en la costa norteafricana, hasta finales del siglo pasado. Se alude a él en otros casos también con el nombre de sabir y, más raramente, con el de petit mauresque.

El estudio de la lingua franca, como el de otros pidgines antiguos, choca inevitablemente con la escasa documentación de que disponemos. Los textos en lingua franca constituyen un repertorio que es, en términos generales, el mismo que ya describió Schuchardt a principios de siglo. Los estudios más recientes no han aportado grandes novedades en este sentido sino que más bien han contribuido a aumentar el número de testimonios y referencias indirectas conocidas.

El conocimiento moderno de la lingua franca se basa fundamentalmente en dos textos de épocas muy diferentes. En primer lugar, disponemos de los datos que proporciona el fraile español Diego de Haedo en su Topographia e Historia general de Argel, de $1612^{2}$. Haedo ofrece información acerca de las condiciones de uso, los hablantes y las características de esta lengua y la acompaña de un número relativamente grande de frases, palabras y conver-

2 Cito por la edición de la Sociedad de Bibliófilos, Madrid, 1927-1929. Hay una completa relación de las noticias que ofrece D. de Haedo sobre la lingua franca en Keith Whinnom, "The Context and Origins of Lingua Franca", en J. Meisel (ed.), Langues en contact, pidgins, créoles, Tübingen, 1977, págs. 3-18. H. Schuchardt proporciona a su vez una lista casi completa de las frases en lingua franca del texto. 
saciones recogidas de boca de árabes y turcos argelinos. Además de la Topographia... de Haedo, disponemos también del Dictionnaire de la Langue Franque ou Petit Mauresque ${ }^{3}$, publicado en Marsella en 1830 para uso de los soldados franceses destinados a Argelia. Este es un documento mucho más amplio y detallado, dispone de un vocabulario de cierta extensión y de un breve repertorio de frases de uso habitual.

Fuera de estos dos testimonios, los textos en lingua franca son pocos y muy breves. De la Edad Media procede el denominado poema de Grion ${ }^{4}$ y frases muy cortas y aisladas en documentos italianos de finales del siglo xv. Del siglo xvi contamos con un villancico de Juan del Enzina y un diálogo en una farsa veneciana de hacia $1550^{5}$. En los siglos siguientes abundan las referencias indirectas $y$ algunas frases en las relaciones de viajes al norte de África, pero también disponemos de un conjunto de materiales de características muy especiales. Se trata de un importante número de diálogos puestos en boca de personajes de procedencia oriental (moros, turcos, griegos, armenios, etc.) presentes en comedias de muy diversa procedencia. Asi, por ejemplo, el habla de ciertos bailarines turcos en Le bourgeois gentilhomme ${ }^{6}$ de Molière, la lengua de personajes levantinos en bastantes comedias de Carlo Goldoni ${ }^{7} \mathrm{y}$, finalmente, la jerga o lengua de los moros del teatro clásico español.

Efectivamente, en el teatro español del Siglo de Oro ${ }^{8}$ se encuentran con mucha frecuencia personajes moros cuyo español está caracterizado por ciertos rasgos fonéticos, morfológicos y sintácticos diferenciales. En realidad, se trata de uno entre varios ejemplos más de variedades de español usadas en

3 La primera noticia de este pequeño diccionario la da, como de casi todo, $\mathrm{H}$. Schuchardt. Además, W. Coates, "The Lingua Franca", en F. Ingemann (ed.), Proceedings of the Vth Kansas Linguistic Conference, Lawrence, Kansas, 1971, págs. 25-34, contiene una copia completa de su segunda parte, la dedicada a las frases de uso corriente.

4 Gustave Grion, "Farmacopea e lingua franca del Dugento", Archivio Glottologico Italiano, 12, 1988, págs. 181-186. Se trata de un poema burlesco que reproduce las frases que una mujer de la isla de Djerba dirige a un italiano. G. Grion lo fecha aproximadamente en la primera mitad del siglo xiv.

5 De la farsa italiana da noticia H. Schuchardt, pág. 75. El poema de Juan del Enzina es comentado en $\mathrm{K}$. Whinnom, pág. 8.

- Molière, Oeuvres complètes, ed. P. A. Touchard, París, 1962. También señalado por H. Schuchardt, pág. 75.

7 Esta jerga empleada por Goldoni aparece descrita y comentada con gran detalle por Henry Kahane y Renée Kahane, "Lingua franca: The Story of a Term", $R P h, 30$, 1976, págs. 25-41.

8 H. Schuchardt cita las obras de Lope de Vega, Vélez de Guevara, Téllez de Acevedo, Cañizares y, sobre todo, Calderón. A. E. Sloman, "The Phonology of Moorish Jargon in the Works of Early Spanish Dramatists and Lope de Vega", Modern Languages Review, 44, 1949, págs.. 207-217, incluye también obras de Gil Vicente, Diego Sánchez de Badajoz, Lope de Rueda y Juan de Timoneda, entre otros. 
el teatro con fines manifiestamente burlescos, entre ellas destacadamente la de negros.

Ya Schuchardt ${ }^{9}$ se refiere con cierto detalle a esta jerga literaria, haciendo notar además cómo era cuidadosamente distinguida de otras de su misma naturaleza. Para Schuchardt se trata de una imitación de la denominada aljamía, entendida como castellano corrompido de uso en la comunicación con moros, acepción que sólo recoge el glosario de Dozy-Engelmann ${ }^{10}$ y que resulta evidentemente casi sinónima de lingua franca. Es esto último y, como veremos después, ciertas características compartidas con los diálogos orientales en Molière y Goldoni, lo que favorece la idea de que el modelo para esta elaboración literaria que es la lengua de moros lo suministró la lingua franca. Es precisamente esta cuestión la que nos proponemos discutir a continuación.

\section{Características de la lengua de moros.}

La variedad de español que hemos venido denominando lengua de moros es una de las más frecuentes en el teatro español de los siglos XVI y xVII para caracterizar a ciertos personajes de procedencia no castellana. Ya en los comienzos de la literatura dramática española se recurre a la lengua con estos fines. Ahí están los pastores sayagueses como ejemplo temprano de este procedimiento ${ }^{11}$. Enseguida vendrán en los primeros años del siglo xvi moros, gitanos y negros ${ }^{12}$, cada uno con una variante peculiar de castellano que con el tiempo constituirá una jerga invariablemente aprovechada por los autores con fines cómicos. De hecho, junto a los moros que hacen papel de graciosos y hablan la jerga, encontramos moros principescos y de ascendencia noble cuyo español resulta intachable. Las particularidades lingüisticas adjudicadas a los moros en las obras de esta primera época permanecen en el teatro del xvir con muy pequeñas variaciones, de tal modo que no resulta difícil establecer un catálogo de las más frecuentes e interesantes. Es precisamente esto lo que hemos hecho nosotros a partir fundamentalmente de la revisión de algunas obras de Calderón de la Barca ${ }^{13}$.

- H. Schuchardt, pág. 76.

10 R. P. Dozy y W. H. Engelmann, Glossaire des mots espagnols et portugais derivés de l'arabe, Leiden, $1896^{2}$.

11 A. E. Sloman, pág. 207, cita la Vita Cristi de fray frígo de Mendoza de 1480 como el primer ejemplo de uso literario de un dialecto de pastores.

12 A. E. Sloman, pág. 208, contiene la lista de los primeros textos en que aparecen estas jergas. Habría que añadir la Comedia llamada Rosabella de M. de Santander, León, 1550 (reimp. facsímil, León, 1989), cuya noticia debo a Luis de Cañigral.

13 Se trata de las siguientes obras: El Gran Principe de Fez, El jardín de Falerina y Amar después de la muerte o el tusani de la Alpujarra (P. Calderón de la Barca, Obras completas, ed. A. Valbuena, Madrid, $1973^{2}$ ). 
El trabajo de Sloman recoge ya con suficiente detalle los rasgos fonéticos más abundantes. Están presentes en casi todos los textos fenómenos como la despalatalización de $[\lambda]$ y de $[n]$, la confusión de vocales y la reducción de diptongos :

(1) a. Alcuzcuz: ¿Qué querer decir aquelio de baril morilio? (Principe, pág. 1369).

b. Zulemilla: Seor crestianilio, osted pase... (Jardin, pág. 1899).

(2) Alcuzcuz: Sonior, si querer sobir... (Principe, pág. 1376).

(3) a. Alcuzcuz: No ser el voneno fuerte; e si es que morer pretendo... (Amar, pág. 371).

b. Alcuzcuz: ¿esto es dormer o morer? (Amar, pág. 372).

(4) a. Alcuzcuz: ... que es morte más dolce. Calderón (Amar, página 371).

b. Zulemilla : ... hacendo no bon mudanza ... (Jardin, pág. 1911).

Menos habituales son la sonorización de oclusivas sordas y el llamado "xexeo", esto es, la pronunciación palatal de la [s] alveolar castellana:

(5) a. Zulema: Ser segreta me embajada ... Lope de Vega (Sloman, pág. 215).

b. Zulemilla: ... que terar como unos berros ... (Jardin, pág. 1898).

(6) a. Moura: mi no xaber que exto extar... Gil Vicente (Sloman, pág. 210).

b. Moro: Ala xaber xiscapara... J. de Timoneda (Sloman, página 210).

Aunque resulten menos emblemáticos del habla de moros, los rasgos morfológicos y sintácticos resultan mucho más interesantes por las relaciones que, como veremos más adelante, permiten establecer con otras variedades lingüisticas de la misma naturaleza.

Dentro de la morfología hay que indicar la confusión de géneros, lo que da lugar a abundantes errores de concordancia:

(7) a. Zulemilla: ; Que el primera luz del sol nos desengañe! (Jardin, pág. 1991).

b. Alcuzcuz: ... que aquí esperar le mandaste con el yegua... (Amar. pág. 373).

Mucho más importantes resultan tres procedimientos sintácticos presentes desde el primer momento. Se trata de la denominada infinitivización, es 
decir, el uso generalizado de las formas de infinitivo en sustitución de las formas flexionadas del verbo, el uso de "estar" con el valor de "ser" y la aparición de formas oblicuas del pronombre en función de sujeto:

(8) a. Moro: Mi xonior, porque llamar? Farsa de los lenguajes (Sloman, pág. 210).

b. Alcuzcuz: Mientras él leer, pus no falta le hacer, ir a ver querría si ganar mi amo o perder por le esperar al venida (Principe, pág. 1386).

(9) a. Zulemilla : ¿Estar acaso cobarde ... que aquí venir a ocultarte? (Jardin, pág. 1899).

b. Alcuzcuz: ... me estar morilio baril que estar vos morazo vejo (Principe, pág. 1371).

(10) a. Alcuzcuz: ... que me saber senda por donde a la cumbre salgas (Principe, pág. 1376). gina 210).

b. Mouro: mi no xaber onde andar... Gil Vicente (Sloman, pá-

Hay que indicar finalmente que, frente a lo que cabria esperar, es el léxico de la lengua de moros el aspecto menos llamativo. Efectivamente, el vocabulario de estos moros del teatro español no es en absoluto diferente del de los personajes que hablan español corriente. No se registra en él presencia de palabras específicas provenientes del árabe ni de ninguna otra lengua, por lo que, en este sentido, la lengua de moros no presenta nada reseñable (cf. supra, ejemplos 1-10).

\section{LENGUA DE MOROS FRENTE A LINGUA FRANCA.}

Los rasgos lingüísticos que muestran los textos en lingua franca más seguros y completos (Haedo y el Dictionnaire) presentan notables diferencias respecto a la lengua de $\operatorname{moros}^{14}$.

Fonéticamente, la lingua franca presenta una indudable base románica en la que aparecen tan sólo modificados con regularidad los diptongos. Están ausentes en la práctica fenómenos como el xexeo y la despalatalización.

La morfología y la sintaxis de la lingua franca son resultado de una reducción profunda de su precedente románico, especialmente visible en la flexión verbal y pronominal. Son casi norma general procesos como la infiniti-

14 Véase H. Schuchardt, W. Coates y B. Collier, "On the Origins of Lingua Franca”, Journal of Creole Studies, 1, 1978, págs. 281-288. 
vización, el uso de pronombres oblicuos en función de sujeto y el uso de estar como cópula universal:

(11) a. Acosi, acosi, mirar como mi estar barbero bono y saber curar, si estar malato (Haedo, II, pág. 106).

b. cosa ti quérir counchar? (Dict., pág. 94).

c. qouesto star véro (Dict., pág. 93).

Pero no son éstas, ni mucho menos, las únicas modificaciones. El artículo prácticamente desaparece:

(12) genti hablar tenir gouerra. Gouerra, con qué natzion? Con francis (Dict., pág. 97).

Junto al infinitivo, usado con valor de presente $y$ futuro, quedan el imperativo y el participio pasado con valor de perfecto:

(13) a. Anda presto piglia, porta fora, guarda diablo ... (Haedo, III, pág. 235).

b. mi non sentito nada (Dict., pág. 97).

Se desarrolla un complemento directo preposicional con per, usado tanto para personas como para cosas:

(14) a. ti conoschir per ellou? (Dict., pág. 96).

b. mi tenir thé mouchou bonou; mi quérir ti goustar per ellou (Dict., pág. 97).

Finalmente, lo más característico de la lingua franca resulta ser el vocabulario. El léxico mayoritario de este pidgin está constituido a partes iguales por palabras españolas e italianas, estas últimas de procedencia dialectal, venecianas, toscanas y meridionales, sobre todo. Junto a este caudal básico y omnipresente, existe un número mucho más limitado de palabras árabes y francesas y algún préstamo occitano, griego, turco, etc. (cf. supra, ejemplos 11-14).

Fuera ya de las características internas de la lingua franca, es muy importante hacer notar que, si bien este pidgin era usado efectivamente por árabes $\mathrm{y}$ turcos, no es menos cierto que era también el medio de comunicación habitual entre los propios europeos confinados en los baños norteafricanos ${ }^{15}$. Cabe pensar pues que, a partir de este uso, se generalizara también fuera de esa situación entre marinos y comerciantes de todo el Mediterráneo y pasara a ser conocido al menos en los países europeos.

15 D. de Haedo, vol. I, cap. XXIX. 
Las diferencias entre la lingua franca y la lengua de moros resultan entonces bien evidentes. En primer lugar, la fonética de la lengua de moros es el componente más singularmente caracterizado de todos con rasgos que tan sólo parcial e irregularmente se dan también en la lingua franca. En segundo lugar, la gramática de la lengua de moros presenta un número menor de rasgos propios, usados además de manera muy poco estable frente a las características gramaticales más estables, complejas y variadas de la lingua franca. Finalmente, la diferencia mayor radica en el léxico, idéntico al del español general en la lengua de moros, de mucha mayor complejidad y variedad en la lingua franca.

A la vista de estos hechos, hay que concluir que la lengua de moros no es en absoluto lingua franca. Se trata de una elaboración literaria construida sobre una base española a partir de rasgos seleccionados como típicos y fácilmente identificables procedentes de otros códigos distintos del español. Los códigos que sirven de inspiración incluyen probablemente dos modalidades lingüisticas distintas pero emparentadas estructuralmente $y$, muy posiblemente, también históricamente, como se puede deducir de sus características. Por un lado, debió de servir de modelo lo que podriamos denominar español con acento árabe, es decir, una interlengua ${ }^{16}$ o lengua de extranjeros. Por otro lado, también más tarde la lingua franca pudo contribuir a la fijación de esta lengua de moros.

La interlengua árabe-española, es decir, la modalidad de español hablado por árabes en un estadio incompleto de aprendizaje, fue sin duda el referente fundamental y mejor conocido de todos los autores españoles. Sin duda, algunos moriscos y los árabes capaces de hablar español constituían un ejemplo vivo de este tipo de lengua. Debió de ser esta modalidad la que proporcionó los rasgos fonéticos básicos de la lengua de moros en la primera mitad del siglo xvI, muy especialmente, el xexeo y la despalatalización, que no se dan en la lingua franca. Hay que notar, al respecto, que los moros del teatro clásico español son en su mayoria moriscos españoles en realidad, lo que avala esta hipótesis.

A partir de finales del xvi y en el xvir el conocimiento de la lingua franca en España tuvo que ayudar a los dramaturgos españoles en la elaboración del tipo de lengua de moros que se observa en Calderón. Los rasgos gramaticales de la lengua de moros, infinitivización, pronombres oblicuos como sujetos y el uso de estar por ser, se verían quizá reforzados por su existencia en la gramática de la lingua franca. Sin embargo, a la vista del léxico exclusivamente español de la lengua de moros, la lingua franca tuvo que ser un modelo muy secundario respecto al anterior, el más inmediato, conocido y

16 John Holm, Pidgins and Creoles, vol. I, Cambridge, 1988, pág. 10. 
reconocible para los españoles de la época. A propósito de esto, resulta decisivo indicar que hechos como la infinitivización son fenómenos casi universales en la parodia literaria y popular de las interlenguas. Del mismo modo, un procedimiento como el uso exclusivo de formas de pronombre régimen es habitual en el español simplificado con el que nos dirigimos a los extranje$\operatorname{ros}^{17}$. En consecuencia, la presencia de ambos hechos en la lengua de moros no es garantía suficiente de la relación de ésta con la lingua franca.

Para concluir este trabajo, creo que es procedente traer a colación aquí un ejemplo paralelo en muchos sentidos a nuestra lengua de moros y que puede servir para confirmar las hipótesis arriba enunciadas. Se trata de la variedad de italiano-veneciano que Goldoni pone en boca de algunos personajes de procedencia oriental en sus comedias ${ }^{18}$. También esta lengua, como indicamos más arriba, ha sido tradicionalmente incluida entre los documentos en lingua franca. Al igual que la lengua de moros es léxicamente español, esta jerga es léxicamente idéntica a la de los personajes italianos. Pero Goldoni ha añadido ciertos rasgos gramaticales peculiares que son exactamente los mismos que están en la lengua de moros: inifinitivización, pronombres oblicuos en función de sujeto y stare por essere:

(15) Isidoro (un corfiota): Star bella; ma da bella tanto mi no importar; so bontà, so modestia, squasi me innamorar ... (Donne, pág. 265) ${ }^{19}$.

Hay que recordar que se conocen ejemplos de interlenguas de base veneciana, el llamado veneciano colonial, en uso durante la Edad Media en la costa griega y dálmata ${ }^{20}$. Fueron probablemente estas modalidades venecianas el modelo de esta "lengua de levantinos" de Goldoni y, como en el caso español, también la lingua franca pudo jugar aqui cierto papel secundario y de refuerzo.

\section{Conclusiones.}

Concluyendo, pues, la lengua de moros del teatro español, y modalidades emparentadas como la "lengua de levantinos" de Goldoni, no constituyen ejemplos de lingua franca. Se trata de una elaboración artificial y burlesca,

17 C. Ferguson, "Absence of Copula and the Notion of Simplicity: A Study of Normal Speech, Baby Talk, Foreigner Talk and Pidgins", en D. Hymes (ed.), Pidginisation and Creolization of Languages, Cambridge, 1971, págs. 141-150.

$18 \mathrm{H}$. Kahane y R. Kahane, describen y comentan esta modalidad que presentan ciertos personajes eslavos, turcos, griegos y armenios en varias de sus comedias: $I$ pettegollezzi delle donne, Le donne di casa soa, La birba, etc.

19 C. Goldoni, Collezione completa delle commedie, Piacenza, 1837 y sigs.

20 Véase $\mathrm{H}$. Kahane y $\mathrm{R}$. Kahane y $\mathrm{N}$. Vianello, "Lingua franca di Barberia e Lingua franca di Dalmazia", Lingua Nazionale, 16, 1955, págs. 67-69. 
para uso literario, construida a partir de la selección de los rasgos más fácilmente reconocibles de una interlengua o "lengua con acento extranjero" de la época, la de árabes y moriscos. Las características de esta jerga del teatro pudieron ser reforzadas posteriormente gracias a la extensión del conocimiento en España de la lingua franca.

\section{Abreviaturas de las fuentes utillzadas.}

Dict. = Dictionnaire de la langue franque ou petit mauresque, Marsella, 1830.

Donne = C. Goldoni, Le donne di casa soa, en Collezione completa delle commedie, Piacenza, 1837 y sigs.

Haedo $=$ Fray D. de Haedo, Topographia e historia general de Argel, 3 vols., Madrid, 1927-1929.

Amar $=$ P. Calderón de la Barca, Amar después de la muerte o el tuzani de la Alpujarra, en Obras completas, ed. A. Valbuena, Madrid, $1973^{2}$.

Jardin = P. Calderón de la Barca, El jardín de Falerina, ibid.

Principe $=$ P. Calderón de la Barca, El Gran Principe de Fez, ibid.

Sloman = A. E. Sloman, "The Phonology of Moorish Jargon in the Works of Early Spanish Dramatists and Lope de Vega", Modern Languages Review, 44, 1949, páginas 207-217. 